\title{
The usefulness of defining type 2 intestinal failure using the 28 days of parenteral nutrition surrogate marker
}

\author{
J. Saunders, T. Smith, A. King and M. Stroud \\ Southampton NIHR Nutrition, Diet and Lifestyle BRU, Southampton General Hospital, Southampton, SO16 6YD, UK
}

Patients with type 2 intestinal failure (IF) are a heterogeneous group as reflected in existing definitions, and this diversity and consequent difficulties in classification can result in problems for audit and data reporting ${ }^{(1)}$. The incidence of type 2 IF is estimated to be 9 patients/ million per year, based on patients who received more than 28 days of parenteral nutrition (PN) and this surrogate marker has become increasingly incorporated into some clinical practice but without evidence as to validity ${ }^{(2)}$.

We undertook a retrospective study to interrogate the PN database in our institution from 2008-2010. Southampton is a large teaching hospital with specialist regional services including an expanding IF service. We identified 70 patients who had received more than 28 days of PN and after detailed notes review, the consensus view of 3 clinicians identified that only $45(64 \%)$ of patients fulfilled the criteria for type 2 IF. 17 (24\%) patients were considered to have type 1 IF with prolonged recovery, predominantly due to age and co-morbidity. This group also included 3 patients with significant complications following bone marrow transplant. 8 (11\%) patients were new patients referred for home PN (type $3 \mathrm{IF}$ ).

\begin{tabular}{lll}
\hline & Type 2 IF & Non Type 2 IF \\
\hline $\mathrm{N}$ & 45 & 25 \\
Male: Female & $26: 19$ & $12: 13$ \\
Mean age (yrs) & $57(17-85)$ & $56(18-85)$ \\
Mean length of stay (days) & $107(30-297)$ & $71(32-211)$ \\
Mean ITU stay (days) & $9(0-63)$ & $1(0-7)$ \\
Mean PN days & $70(28-184)$ & $44(28-80)$ \\
Enterocutaneous fistulae & $55.6 \%$ & $0 \%$ \\
Laparostomy & $29 \%$ & $0 \%$ \\
Hospital mortality & $6.6 \%$ & $8 \%$ \\
\hline
\end{tabular}

During this period the Southampton IF team also established a regional clinical network to aid development of specialist IF services. As a consequence there has been a marked increase in the number of patients referred specifically for management of type 2 IF. In the patients with type 2 IF 29 were regional referrals, of which 12 (41\%) were specifically for IF management (8 patients in 2010 , 4 in 2009 and 0 in 2008). It is acknowledged that not all patients around the region who receive more than 28 days of PN or that have type 2 IF are referred. These figures are therefore likely to represent a significant underestimate of the prevalence of type 2 IF at a regional level. We also reviewed all patients with type 2 IF in 2010, identifying 6 patients who received less than 28 days of PN who were discharged with a combination of complex parenteral fluids and enteral feeding.

This study demonstrates that the "more than 28 days of PN" criterion is not a specific or sensitive method for identifying patients with type 2 IF. However, it may be a useful marker of disease severity and the need for higher levels of resources and therefore be of value in determining regional funding mechanisms. It also shows that regional IF centres currently perform a significant volume of unfunded specialist work and that forming clinical networks can help develop local IF services.

1. Schaffer J (2002) Intestinal failure: definition and service development. Clin Nutr 21 (suppl. 1), 144-5.

2. NHS specialised Services. A strategic framework for intestinal failure and home parenteral nutrition services for adults in England. April 2008. www.specialisedservices.nhs.uk 